\title{
DocuBits and Containers: Providing e-Document Micro-mobility in a Walk-Up Interactive Tabletop Environment
}

\author{
Katherine Everitt, Chia Shen, Kathy Ryall, and Clifton Forlines \\ Mitsubishi Electric Research Laboratories, 201 Broadway, Cambridge, MA, USA \\ everitt@cs.washington.edu, \{shen, ryall, forlines\}@merl.com
}

\begin{abstract}
A key challenge in supporting face-to-face collaborative work is edocument micro-mobility: supporting movement of digital content amongst shared display surfaces and personal devices at arbitrary levels of document granularity. Micro-mobility is a dexterity that physical paper artifacts afford the ability to be handled with any position and placement, to be dismantled, cut and torn apart, marked up, reassembled and sorted. To support micromobility for electronic content and group work, we propose DocuBits and Containers. DocuBits offer the metaphor of a paper-cutter and a scanner for electronic documents. A portion of screen 'bits' from any application or any parts of visible display can be cut, grabbed, sent and launched onto a different display surface or device with minimal interaction - merely three mouse/stylus click-select. Once arrived on the target display surface, DocuBits can be arbitrarily positioned, re-oriented, marked up, and pulled into other documents, or again sent to other display surfaces. A Container is a composite draft of DocuBits and other documents, usually composed as the outcome of a collaborative meeting.
\end{abstract}

\section{Introduction}

When collaborating in a face-to-face setting, people often use large surfaces such as tables as the physical location for paper-based information, making information easily viewable and accessible by all. In contrast, electronic documents (edocuments) are usually located on devices designed for individual use, accessed by single user input mechanisms such as mice and keyboards. Today's e-documents do not support dynamic mobility-related techniques such as decomposition, on-the-spot sorting or piling - actions typical in many group settings such as brainstorming sessions or content design and creation meetings where the electronic artifacts to be used are often brought in by each participant; groups then work together with edocuments in-situ to create composite draft documents. In such multi-device environments, e-documents lack some of the basic capabilities of their paper (physical) counterparts.

Paper documents afford the ecological dexterity [2] of re-orientation, markup, ease of passing amongst participants, and even the option of tearing off of part of a page of a document so it can be re-arranged and reassembled with respect to other pages. E-documents lack support for this type of interaction and composition. Current interactive tabletop and roomware such as $[5,6,7]$ do not provide the 
necessary micro-mobility of artifacts (as paper documents do) - it is often not possible to move arbitrary portions and granularities (e.g., a line, a word or a section of a screen) of digital data in a simple yet holistic interaction style across device boundaries. Most current systems support only whole document movement amongst display devices.

Some recent work touches on micro-mobility of documents in a desktop setting. WinCuts [8] offers a mechanism to carve out any portion of an active window for spatial organization of information. WinCuts are synchronized with the original content, and so support alternate visual presentation but not version comparison. Synchronizing Clipboards [3] allow two computers to share a system clipboard. This supports mobility of files and ASCII text in an "invisible" fashion, and is best suited to a single user with multiple machines. ScreenCrayons [4] collects annotations of screen captures and provides capability to annotate, classify, and highlight notes. It is designed for use by a single user on a desktop machine.

In this paper, we present our initial design and prototype of Docubits in an interactive walk-up multi-user tabletop environment [1, 6], as shown in Fig. 1 (left).

\section{DocuBits and Containers: Design and Prototype}

DocuBits mechanisms allow users to easily carve out any portions of documents or any portion of visible screen display and operate with them as full documents. We support the amalgamation of documents and DocuBits into composite documents, called "Containers," which are saved as HTML for easy after-meeting portability.

DocuBits: Figures 1, 2 and 3 illustrate the sequence of interaction of Docubits creation and movement. A DocuBit is a portion of the visible screen, including both the content and its layout in the workspace. A portion of screen from any application or arbitrary portion of the visible display can be cut, grabbed, sent and launched onto a different display surface or device. We currently support two forms for the captured DocuBits: images and editable text, making DocuBits portable to any platform. DocuBits provide a quick and fluid way for multiple people to bring their own draft documents or bits and pieces of ideas onto the meeting table, and can be used to clarify spatial relationships between content, highlight a point, and remove private information. They can represent many levels of granularity: from a word or a section of a window, to an entire window or even the spatial relationships between windows.

DocuBits are designed to be lightweight, and easy to create and send. The interaction in creating DocuBits approximates WYSIWYG; users select any region from what can be seen and immediately make it into a new DocuBit. When the user starts a new visual DocuBit by clicking the "New Screen Section" button (Fig 1 right), a screenshot is taken and the user selects the relevant screen subsection with a rectangular selection gesture (Fig 2 left). Text-based DocuBits use the system clipboard in the same way as conventional text copy. DocuBits appear in Java frames, and are sent between devices using sockets. A preview window is provided with three buttons: "Send", "Discard" and "Save" (Fig 2 right), to allow the user to easily cancel, save a copy, and/or send the DocuBit to a different display surface. DocuBits are not tethered to the original documents or screen content. As they are not synchronized with other versions of the same content, but are effectively a 
snapshot in time, they can be compared to each other like different paper drafts. Each DocuBit stores meta-data about its creation location and time. This allows users to later determine when and where the DocuBit was created. They can easily be reorganized using the Container described below. Both DocuBits and Containers are implemented in Java.
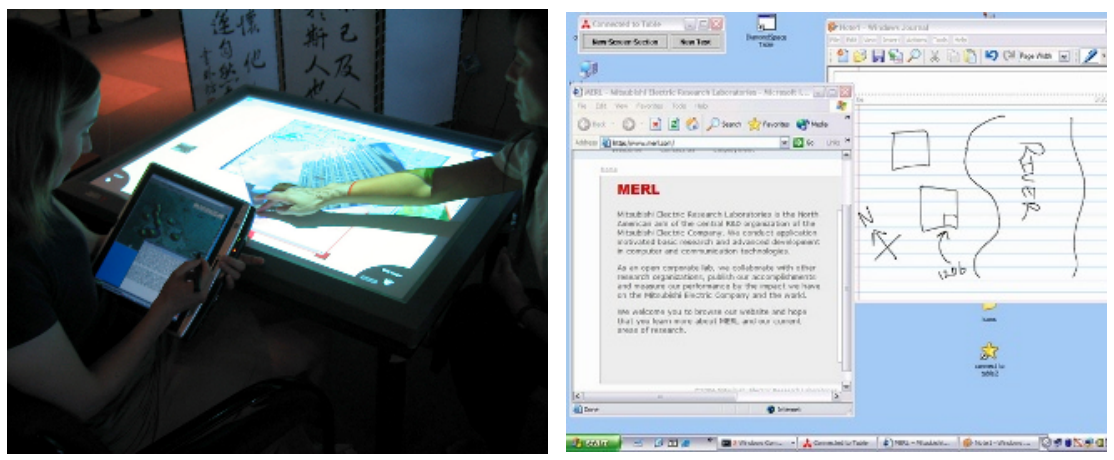

Fig. 1. Sample Setup: Left: Meeting around an interactive UbiTable [6] tabletop. Right: TabletPC desktop screen with "New Screen Section" button on top left of desktop display.
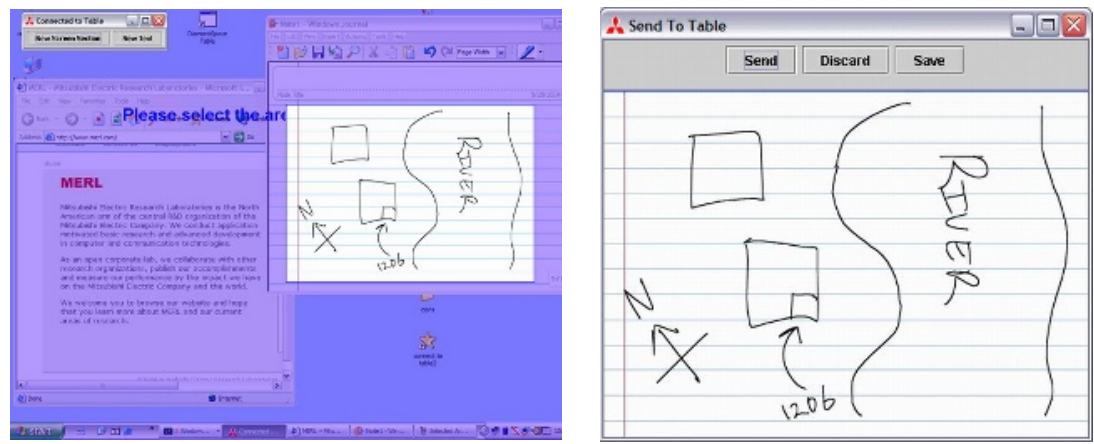

Fig. 2. "New Screen Section" button grays out the screen: Left: User selects a portion of screen area which becomes un-grayed. Right: Selected screen bits automatically become a DocuBits preview window with three buttons (Send, Discard, Save).

Containers: A container object supports micro-mobility by providing a mechanism to recombine objects. We have prototyped the Container in two forms: as a list of objects and a more flexible collage of items. A user can "drop" an object into the Container list by sliding an object over it and releasing. Containers can be copied, deleted, and moved between the display surfaces; in essence they are a new e-document. When saved on a personal device, a Container, in the form of an HTML document with meta-data, contains a record of where the pieces came from, the document type of each piece, and could also contain information about who edited which parts. This can be easily viewed later on other devices.

Linking Devices: Portable devices (e.g., laptops or TablePCs) can be linked to the table on-the-fly using portals similar to those used in [6]. This creates a virtual 
link between the corner on the table closest to the device's user and the device (see Fig 3). Objects dragged onto a portal will be copied and a representation will appear on the linked device. DocuBits can be moved between devices in this way.

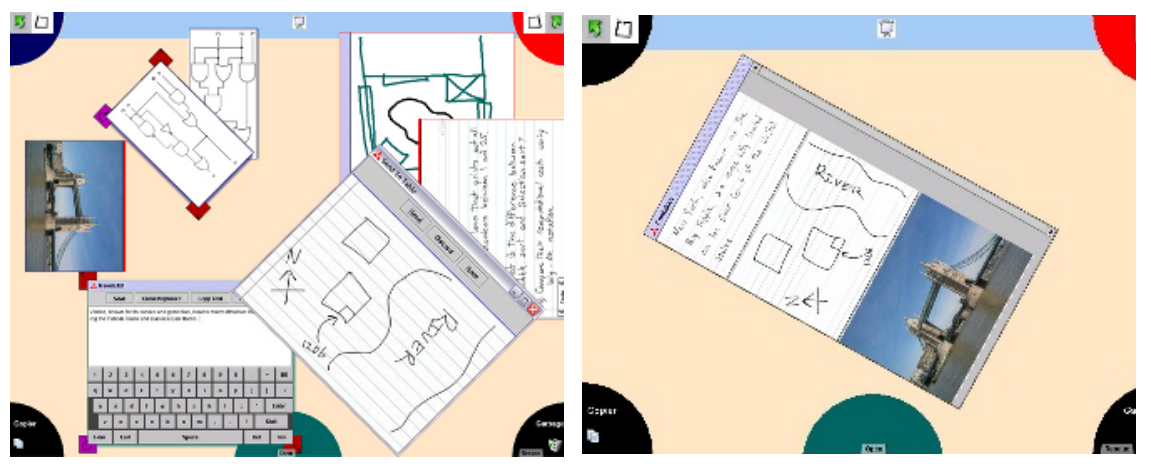

Fig. 3. On the TableTop: Left: Screenshot of a tabletop with DocuBits. Corners of the tabletop are wormhole portals to personal devices. Right: DocuBits are combined into a Container on the tabletop comprised of a diagram, some text and an image.

\section{Conclusions and Future Work}

DocuBits raise a number of interesting directions for future work. We would like to explore different usage patterns, expand selection beyond rectangular boxes, and explore the idea of synchronization of items. Synchronization could potentially improve speed of collaboration but might also cause confusion with so many objects and increase difficulty of document comparison. The question is still open as to whether making the system functionally more sophisticated in this way would benefit the user or would merely make the system overwhelmingly complex.

\section{References}

1. Everitt, K., Forlines, C., Ryall, K., Shen, C., "Observations of a Shared Tabletop User Study," Interactive Poster, ACM CSCW 2004.

2. Luff, P., Heath, C., "Mobility in Collaboration,". Proc. ACM Computer Supported Cooperative Work (CSCW), 1998. pp. 305-314.

3. Miller, R., Myers, B., "Synchronizing Clipboards of Multiple Computers," Proc. ACM UIST 1999.

4. Olsen, D., Taufer, T., Fails, J.A., “ScreenCrayons: annotating anything,” Proc. ACM UIST 2004.

5. Rekimoto, J., Saitoh, M., “Augmented Surfaces: A Spatially Continuous Work Space for Hybrid Computing Environments," Proc. ACM CHI 1999.

6. Shen, C., Everitt, K.M.; Ryall, K., "UbiTable: Impromptu Face-to-Face Collaboration on Horizontal Interactive Surfaces,” Proc. UbiComp 2003. LNCS 2864. 281-288.

7. Streitz, N.A., Tandler, P., Muller-Tomfelde, C., Konomi, S. "i-LAND: An Interactive Landscape for Creativity and Innovation," Proc. ACM CHI 1999.

8. Tan, D.S., Meyers, B., Czerwinski, M. "WinCuts: Manipulating Arbitrary Window Regions for More Effective Use of Screen Space," Extended Abstract, ACM CHI 2004. 